\title{
Analysis of National Drug Code Identifiers in Ambulatory E-Prescribing
}

\author{
Ajit A. Dhavle, PharmD, MBA; Stacy Ward-Charlerie, PharmD; Michael T. Rupp, PhD;
} Vishal P. Amin, PharmD; and Joshua Ruiz, CPhT

\begin{abstract}
BACKGROUND: Communication of an accurate and interpretable drug identifier between prescriber and pharmacist is critically important for realizing the potential benefits of electronic prescribing (e-prescribing) while minimizing its risk. The National Drug Code (NDC) is the most commonly used codified drug identifier in ambulatory care e-prescribing, but concerns have been raised regarding its use for this purpose.
\end{abstract}

OBJECTIVES: To (a) assess the frequency of NDC identifier transmission in ambulatory e-prescribing; (b) characterize the type of NDC identifier transmitted (representative, repackaged, obsolete, private label, and unit dose); and (c) assess the level of agreement between drug descriptions corresponding to NDC identifiers in electronic prescriptions (e-prescriptions) and the free-text drug descriptions that were entered by prescribers.

METHODS: We analyzed a sample of 49,997 e-prescriptions that were transmitted by ambulatory care prescribers to outlets of a national retail drugstore chain during a single day in April 2014. The First Databank MedKnowledge drug database was used as the primary reference data base to assess the frequency and types of NDC numbers in the e-prescription messages. The FDA's Comprehensive NDC Standard Product Labeling Data Elements File and the National Library of Medicine's RxNorm data file were used as secondary and tertiary references, respectively, to identify NDC numbers that could not be located in the primary reference file. Three experienced reviewers compared the free-text drug description that had been entered by the prescriber with the drug description corresponding to the NDC number from 1 of the 3 reference database files to identify discrepancies. Two licensed pharmacists with residency training and ambulatory care experience served as final adjudicators.

RESULTS: A total of 42,602 e-prescriptions contained a value in the NDC field, of which 42,335 (84.71\%) were found in 1 of the 3 study reference databases and were thus considered to be valid NDC numbers. A total of $28,172(67.70 \%)$ e-prescriptions in the sample were found to contain a representative NDC number, according to the definition used by the National Council for Prescription Drug Programs (NCPDP). The remaining e-prescriptions consisted of 4 subtypes of unrepresentative NDC numbers. In 41,298 (97.55\%) e-prescriptions that contained an NDC number, the drug description associated with the number from 1 of the 3 data source files pointed to the identical semantic drug concept as the free-text drug description that had been entered by the prescriber. However, in $87(0.21 \%)$ e-prescriptions, the free-text drug descriptions and the drug description associated with the NDC number pointed to completely different semantic drug concepts.

CONCLUSIONS: We found the use of NDC identifiers in our sample of e-prescriptions to be relatively high. However, approximately one-third consisted of unrepresentative NDC numbers (obsolete, repackaged, unit dose, or private label) that have the potential to create workflow disruptions at the dispensing pharmacy. Most disturbing was our finding that more than 2 out of every 1,000 e-prescriptions in our sample contained a free-text drug description that pointed to a completely different drug concept than that associated with its NDC value. Our study suggests the need for e-prescribing technology vendors to maintain accurate and up-to-date drug database files within their systems and to conduct regular validation checks to ensure that the drug descriptions associated with the NDC identifier and the freetext drug description that is sent in the e-prescription message point to the same drug concept. The FDA may need to consider a more active role in ensuring the accuracy of NDC assignment by drug manufacturers.

J Manag Care Spec Pharm. 2015;21(11):1025-31

Copyright $\odot 2015$, Academy of Managed Care Pharmacy. All rights reserved.

\section{What is already known about this subject}

Most of the more than 1 billion e-prescriptions issued each year in the ambulatory care setting are transmitted through Surescripts' clinical health information network using the NCPDP SCRIPT standard.

National Drug Code (NDC) numbers are the predominant drug identifiers used in ambulatory care e-prescribing in the United States.

Concerns about discrepancies between the free-text drug description and that implied by the NDC number have been expressed by prescribers and pharmacists.

\section{What this study adds}

The use of NDC identifiers in ambulatory e-prescribing is quantified. The proportion of valid and representative NDCs in ambulatory e-prescriptions is distinguished.

The incidence and nature of discrepancies that exist between the free-text drug descriptions and the drug descriptions implied by NDCs are identified.

E lectronic prescribing (e-prescribing) can enhance the quality of patient care and improve prescriber and pharmacy productivity and workflow. ${ }^{1-4}$ Realizing the full potential of this transformative technology, however, continues to be a work in progress as key challenges remain. ${ }^{5}$ Core among these is to ensure that the e-prescription message communicates a complete and unambiguous drug identity to receiving pharmacies that can be accurately interpreted by pharmacists and their computer systems. ${ }^{5-8}$ 
Virtually all electronic prescriptions (e-prescriptions) issued in the ambulatory care setting in the United States use the SCRIPT standard, a message format maintained by the National Council for Prescription Drug Programs (NCPDP). The most widely used version of the SCRIPT standard, version 10.6, requires only the inclusion of a free-text drug description in the Drug segment of the message. While often transmitted concurrently with the free-text drug description, supplemental codified drug identifiers are not currently required for successful transmission of the e-prescription message. ${ }^{9}$

The 1972 Drug Listing Act requires U.S. registered drug manufacturers to provide the U.S. Food and Drug Administration (FDA) with a current list of all drugs manufactured and commercially distributed along with their unique National Drug Code (NDC) identifiers..$^{10}$ Originally developed for Medicare outpatient drug reimbursement, NDC identifiers have subsequently been used for a variety of other purposes, including e-prescribing. ${ }^{11,12}$ But while the NDC is currently the primary codified drug identifier used in e-prescribing, concerns have been raised regarding its adequacy for this purpose, and alternatives have been proposed. ${ }^{7,13}$ In particular, concerns have been raised about the absence of an authoritative and up-to-date repository to identify and cross-reference NDC identifiers. ${ }^{5,7,13}$ Although the FDA publishes manufacturersubmitted NDC numbers and corresponding drug information in the NDC Directory, this source has been criticized for being inaccurate and out-of-date due to incomplete submissions by manufacturers and delays in the entry of data. ${ }^{7,11}$

By definition, each NDC number is specific to a particular manufacturer/labeler, product, and associated packaging information. As a result, a single drug description concept (i.e., drug name, strength, and dosage form) may have multiple NDC numbers, which makes the NDC cumbersome and potentially confusing to implement in e-prescribing systems. ${ }^{11,14}$

In response to this recognized problem, the e-prescribing industry has adopted use of the "representative NDC number" in all e-prescribing transactions. The NCPDP's SCRIPT Implementation Recommendations document describes a representative NDC number as an 11-digit value that is intended to represent a category of medication irrespective of the package size and the manufacturer or labeler, thereby allowing the pharmacist flexibility and discretion to select the preferred product for a given patient. ${ }^{15}$ A representative NDC number should be assigned only to a nationally available product, and it should not be 1 of 4 types of unrepresentative NDC numbers; i.e., repackaged, obsolete, private label, or unit dose. ${ }^{15}$

If a representative NDC identifier is included in an e-prescription message, it is transmitted in the Drug Product Code field. If this field is populated, the SCRIPT standard also requires inclusion of a drug product code qualifier that indicates the type of identifier that is being transmitted (e.g., NDC, Universal Product Code, etc.). When a representative NDC identifier or other drug identifier is transmitted, it is essential that the free-text drug description associated with the drug identifier and the required free-text drug description string that was entered or selected by the prescriber point to the same drug concept. ${ }^{5,13,15}$

In practice, e-prescribing software applications often add an NDC or other drug identifier to the e-prescription message on the "back end" before it is transmitted to the pharmacy, sometimes without the knowledge of, or approval by, the prescribing clinician. Moreover, although the standard of practice dictates that pharmacists should review the free-text drug description that was selected or entered by the prescriber, some pharmacy computer systems prepopulate pharmacists' display screens with the drug description that is linked to the NDC identifier or other drug identifier to facilitate efficient processing.

The objectives of this study were to (a) assess the frequency of NDC identifier transmission in ambulatory e-prescribing; (b) characterize the type of NDC identifier transmitted (representative, repackaged, obsolete, private label, and unit dose); and (c) assess the level of agreement between drug descriptions corresponding to NDC identifiers in e-prescriptions and the free-text drug descriptions that were entered by prescribers.

\section{Methods}

\section{Design and Data Source}

Data for the study consisted of a random sample of e-prescriptions that were dispensed from pharmacies of a national retail drugstore chain on Monday, April 21, 2014. A Structured Query Language database query was used to randomly extract a sample of e-prescription records from the master pharmacy relational database repository. Data elements extracted for analysis included (a) individual pharmacy store identifier; (b) unique prescriber identifier; (c) free-text drug description; and (d) NDC value and corresponding qualifier value. Extracted data were de-identified by the pharmacy chain as defined by the Health Insurance Portability and Accountability Act Privacy Rule at 45 CFR \$164.514 before they were provided to the research group for analysis. Prescriber identifiers were available only as unique numeric values. No clinical or demographic patient data were made available to the investigation team.

Most physician and pharmacy e-prescribing technology vendors use one of several commercially available drug database compendia for their clinical terminology needs. ${ }^{5}$ The primary reference drug compendium used for this study was First Databank. Widely used by the e-prescribing industry and government agencies, the First Databank drug database includes an NDC attributes table that was used to distinguish and classify the different types of NDC numbers observed in the data sample. . $2,16,17^{-17}$ 


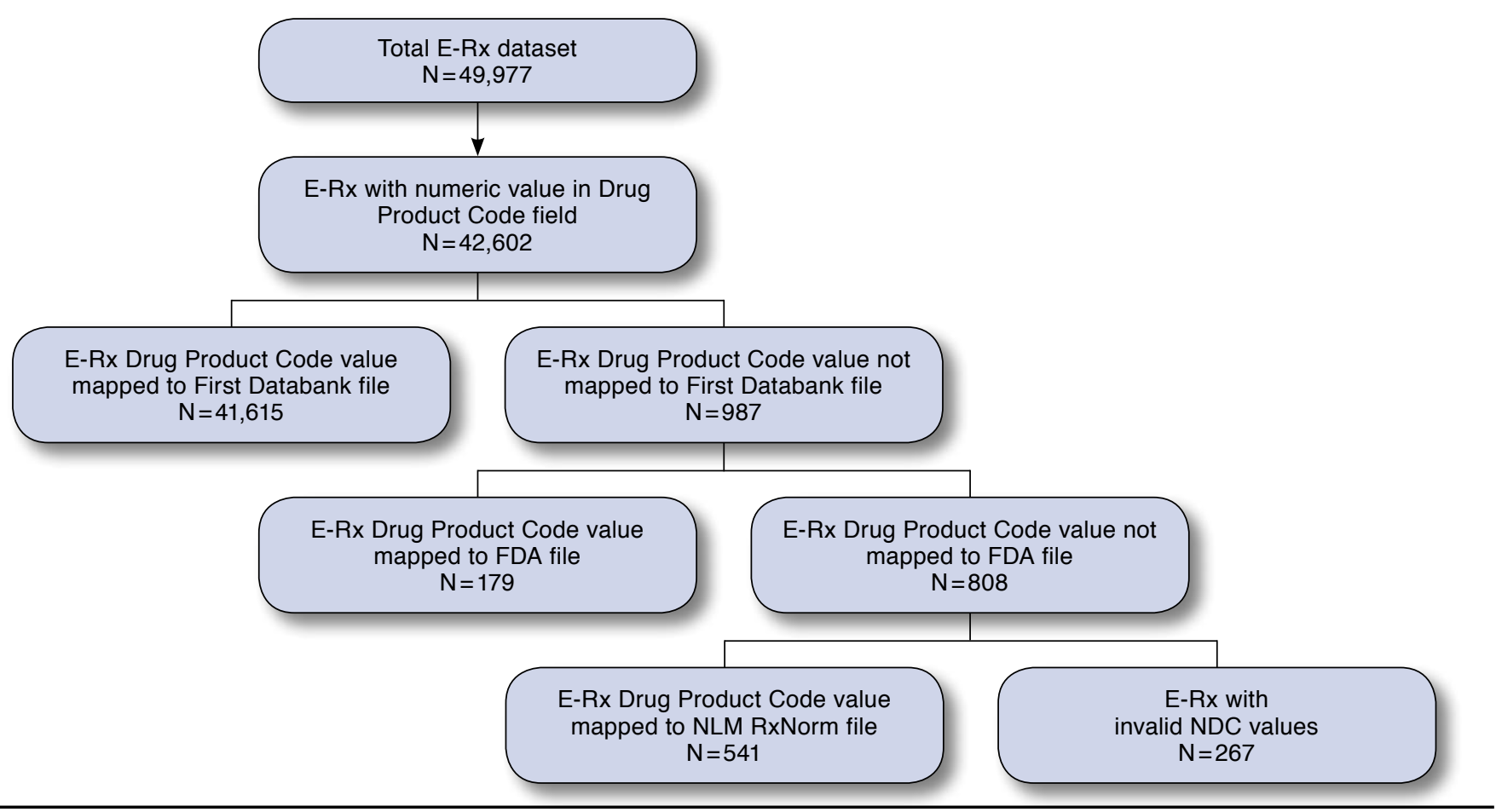

E-Rx=e-prescriptions; FDA =U.S. Food and Drug Administration; First Databank= primary drug compendium used for this study; NDC=National Drug Code; NLM RxNorm = National Library of Medicine drug database.

In theory, the FDA's NDC Directory should include all drug products and corresponding NDC numbers that are manufactured and commercially distributed in the United States. We therefore used it as a secondary source to identify NDC numbers that could not be identified using the First Databank database.

In an effort to be exhaustive in attempting to match e-prescription NDC numbers, we used the National Library of Medicine's (NLM) RxNorm drug database as a tertiary source to identify any remaining NDC numbers that we were unable to match in either First Databank or the FDA's NDC Directory. The RxNorm database receives NDC data from 5 different commercial and government sources, including Gold Standard Drug Database, Multum MediSource Lexicon, FDA Structured Product Labeling (SPL), First Databank National Drug Data File (NDDF) Plus Source Vocabulary, and Veterans Health Administration National Drug File. ${ }^{18}$

Thus, the 3 reference drug database source files that were used in this study were (a) First Databank's April 22, 2014, MedKnowledge (formerly NDDF Plus); (b) the July 7, 2014, FDA Comprehensive NDC SPL Data Elements File; and (c) the April 7, 2014, RxNorm release from the NLM. These were the most current versions of the database reference files available to the researchers at the time of this data analysis and review.

\section{Incidence and Type of NDC Numbers in E-Prescriptions}

All e-prescriptions in the study sample that contained a value in the Drug Product Code field and an "ND" value (denoting NDC) in the corresponding Qualifier field were identified. An attempt was then made to match the NDC number in each e-prescription to the identical code listed in each of the 3 compendia, beginning with the First Databank file. If a match was not found in First Databank, the FDA file was next searched to identify a match. If a match with the primary (First Databank) or secondary (FDA) file failed, the RxNorm file was searched to match remaining NDC numbers. The First Databank reference file also contained attributes that allowed the research team to classify NDC numbers as either representative or 1 of 4 unrepresentative subtypes for analyses. Because a similar NDC attributes table was not available in either the FDA or RxNorm reference files, NDC numbers that could not be identified using the First Databank file were not further subtyped.

\section{Accuracy of Representative NDC Numbers in E-Prescriptions}

Three members of the Surescripts Clinical Quality Team independently reviewed each e-prescription in the sample that contained an NDC number and compared the free-text drug description to that of the drug description corresponding to 
TABLE 1 NDC Numbers Found in First Databank File

\begin{tabular}{|c|c|}
\hline NDC Type & N (\%) \\
\hline Representative NDC numbers & 28,172 \\
\hline $\begin{array}{l}\text { Repackaged NDC numbers } \\
\text { Assigned by a repackager organization. }{ }^{a}\end{array}$ & 6,622 \\
\hline $\begin{array}{l}\text { Obsolete NDC numbers } \\
\text { Discontinued, no longer marketed, no longer produced, no longer distributed, or otherwise made unavailable to the marketplace per } \\
\text { manufacturer or FDA notification. }{ }^{\text {a }}\end{array}$ & $4,182 \quad(10.05)$ \\
\hline $\begin{array}{l}\text { Unit dose NDC numbers } \\
\text { Associated with a product that is packaged in individual unit doses. }{ }^{\text {a }}\end{array}$ & 2,239 \\
\hline $\begin{array}{l}\text { Private label NDC numbers } \\
\text { Associated with products that are labeled for exclusive distribution by specific businesses; these are usually store brand nonprescription } \\
\text { products sold at retail pharmacies. }{ }^{\text {a }}\end{array}$ & $(0.96)$ \\
\hline Total e-prescriptions containing NDC numbers in First Databank file & 41,615 \\
\hline
\end{tabular}

the NDC number from the reference database file that was used to identify it. Each of the reviewers is a certified pharmacy technician with more than 3 years of experience interpreting and processing e-prescriptions in the community practice setting. All had completed training under the supervision of the principal investigator to ensure proficiency.

In cases where consensus was not achieved after independent review, the 3 reviewers discussed the e-prescription as a group in an attempt to reconcile differences. Two secondary reviewers-both licensed pharmacists with ambulatory care experience and residency training — served as final adjudicators in cases in which the 3 primary reviewers could not reach a consensus after discussion. The kappa coefficient using Light's approach for 3 reviewers was calculated to assess interrater agreement between reviewers. ${ }^{19}$

\section{Results}

The final sample used in the analysis consisted of 49,977 e-prescriptions issued by 37,801 community-based prescribers practicing in all 50 U.S. states and the District of Columbia. Prescribers used 519 different e-prescribing software applications to generate e-prescriptions in the sample. E-prescriptions in the sample had been transmitted to 7,391 unique pharmacy store locations of a national drugstore chain.

\section{NDC Identifier Incidence Rate}

As illustrated in Figure 1, 42,602 (85.24\%) e-prescriptions in the sample contained a numeric value in the Drug Product Code field. Of these, all but 1 had a corresponding "ND" qualifier value in the corresponding Drug Product Code Qualifier field, thereby indicating an NDC number. Four e-prescriptions did not contain a numeric value in the Drug Product Code field but included only a qualifier value in the Drug Product Code Qualifier field.
Of the 42,602 e-prescriptions that contained a numeric value in the Drug Product Code field, 41,615 (97.68\%) were matched to an NDC number from the First Databank source file. An additional 179 (0.21\%) were matched to NDC numbers in the FDA file, and 541 (1.27\%) of the remaining e-prescriptions were matched to NDC numbers in the RxNorm file. Thus, a total of 42,335 (84.71\%) e-prescriptions in the original study sample were found to have valid NDC values. The remaining 267 (0.63\%) e-prescriptions did not have numbers with a corresponding NDC match in any of our 3 reference databases, so those numbers were determined to be "invalid" NDC numbers.

\section{Quality of NDC Identifiers in E-Prescriptions}

Using the NDC attributes table available through First Databank, the 41,615 NDC values found in the First Databank file were classified by type (Table 1). A total of 28,172 (67.70\%) e-prescriptions in the sample were found to contain representative NDC numbers. The remaining 13,443 (32.30\%) NDC values were determined to be nonrepresentative NDC numbers.

Most drug compendia maintain a list of obsolete NDC numbers for record keeping and reference purposes. Patient medication histories in e-prescribing software systems sometimes contain obsolete NDC numbers that may be used during e-prescribing. Although the FDA recommends that drug manufacturers wait a minimum of 5 years before reusing old NDC numbers, this guidance is not strictly followed, and 2 distinctly different drug products may have the same NDC identifier. ${ }^{20}$ Repackaged drug and private label manufacturers have been known to arbitrarily assign or reuse NDC numbers, which are also considered nonrepresentative by the NCPDP. ${ }^{20}$

Of the 13,443 nonrepresentative NDC numbers, 6,622 were classified as repackaged NDC numbers, 4,182 as obsolete NDC numbers, 2,239 as unit dose NDC numbers, and 400 as private label NDC numbers. 


\section{TABLE 2 Agreement Between Free-Text Drug} Descriptions and NDC Descriptions

\begin{tabular}{|c|c|}
\hline Level of Agreement & $\mathrm{N}(\%)$ \\
\hline $\begin{array}{l}\text { Drug descriptions were matched to the identical } \\
\text { semantic drug concept. } \\
\text { Example: Amoxicillin } 500 \mathrm{mg} \text { capsule and Amoxicillin } 500 \mathrm{mg} \\
\text { oral capsule }\end{array}$ & $41,298(97.55)$ \\
\hline $\begin{array}{l}\text { Drug description differences were limited to brand } \\
\text { versus generic differences. } \\
\text { Example: Lipitor } 10 \mathrm{mg} \text { oral tablet and atorvastatin calcium } \\
10 \mathrm{mg} \text { oral tablet }\end{array}$ & $752 \quad(1.78)$ \\
\hline $\begin{array}{l}\text { Drug description differences were limited to unit dose } \\
\text { package differences. } \\
\text { Example: Zithromax pack versus Zithromax } 250 \mathrm{mg} \text { tablet }\end{array}$ & $149 \quad(0.35)$ \\
\hline $\begin{array}{l}\text { Drug descriptions pointed to completely different } \\
\text { semantic drug concepts. } \\
\text { Example: Tramadol HCL } 50 \mathrm{mg} \text { tablet versus Tramadol HCL } \\
\text { ER } 300 \mathrm{mg} \text { tablet }\end{array}$ & $87(0.21)$ \\
\hline $\begin{array}{l}\text { Drug description differences were limited to brand } \\
\text { versus brand differences. } \\
\text { Example: Prozac } 20 \mathrm{mg} \text { capsule versus Selfemra } 20 \mathrm{mg} \text { capsule }\end{array}$ & $29 \quad(0.07)$ \\
\hline $\begin{array}{l}\text { Drug descriptions were not complete and could not be } \\
\text { assessed. } \\
\text { Example: Levaquin tablet versus Levaquin } 250 \mathrm{mg} \text { oral tablet }\end{array}$ & $20 \quad(0.05)$ \\
\hline Total & 42,335 \\
\hline
\end{tabular}

HCL ER = hydrochloride extended release; $m g=$ milligrams.

\section{Agreement of NDC Descriptions with Free-Text Drug Descriptions}

As illustrated in Table 2, 41,298 (97.55\%) e-prescriptions that contained an NDC number had a drug description associated with the NDC number that pointed to the identical semantic drug concept as the free-text drug description that had been entered by the prescriber. In 752 (1.78\%) instances, mismatches between the free-text drug description and the NDC-associated drug descriptions were limited to brand versus generic distinctions for the same drug ingredient. In $29(0.07 \%)$ instances, the dissimilarities between the free-text drug descriptions and the NDC-associated drug descriptions were due to differences in the drug brand name only.

There were 149 (0.35\%) e-prescriptions where the drug description differences were due to unit dose package variances. In 87 (0.21\%) e-prescriptions, the free-text drug description and the drug description associated with the NDC number pointed to completely different semantic drug concepts. Finally, the free-text drug descriptions sent in 20 (0.05\%) e-prescriptions were incomplete and could not be assessed.

\section{Discussion}

To our knowledge, this is the first rigorous empirical investigation of NDC usage in e-prescribing in the ambulatory practice setting. As expected, we found the use of NDC identifiers in our sample e-prescriptions to be relatively high at slightly more than 85\%. Moreover, all but 267 (0.63\%) NDC numbers in the assessed e-prescriptions were considered to be "valid," insofar as they could be successfully mapped to an identical NDC value in 1 of 3 source compendia that were used in the study, albeit not necessarily to the same drug concept.

Surprisingly, only $67.7 \%$ of e-prescriptions that contained an NDC number included a value considered to be a representative NDC number, according to the definition used by NCPDP. The remaining 32.3\% were considered to be unrepresentative and were further classified by NDC subtype as obsolete, repackaged, unit dose, or private label NDC numbers. This finding is noteworthy because receipt of an unrepresentative NDC number by a dispensing pharmacy can cause workflow disruptions or physician callbacks, or can even result in a patient safety threat if the number is not in the receiving pharmacy's system or is associated with a different product. It is for this reason that NCPDP recommends that a repackaged, obsolete, private label, or unit dose NDC number not be used in e-prescribing unless it is the only NDC number available to identify that category of medication. ${ }^{15,20}$ Incorrect implementation of drug compendia drug identifiers by the e-prescribing technology vendors and the lack of regular updates to the drug database files by them or at their individual end-user practice sites may explain some of these observed data discrepancies.

In this study, $97.55 \%$ of e-prescriptions with valid NDC numbers had drug descriptions that matched to identical semantic drug concepts. The remaining 2.45\% contained some level of mismatch. Our comparison of (required) free-text drug descriptions entered by prescribing clinicians and the drug description corresponding to (optional) NDC numbers that were concurrently transmitted in the e-prescription message supports previous research and substantial anecdotal reports from pharmacists regarding discrepancies between drug identifiers and free-text drug descriptions in e-prescriptions. ${ }^{21-25}$

Some e-prescribing systems allow users to enter free-text drug descriptions or modify drug compendia/e-prescribing technology vendor-preferred drug description values upon selection, and industry-wide standardization of drug description is still lacking. ${ }^{5}$ Our results indicate that $0.05 \%$ of the e-prescriptions contained a drug description that was incomplete and hence could not be accurately compared with the NDC-associated drug description.

Perhaps most disturbing was the finding that more than 2 e-prescriptions per thousand $(0.21 \%)$ contained a free-text drug description that pointed to a completely different drug concept than that associated with its NDC number. Such discrepancies constitute a significantly greater threat to patient health and safety if not caught by the pharmacy staff or the pharmacist during the prescription fulfillment process than a mere brand versus generic or brand versus brand mismatch.

There are several possible explanations for discrepancies we observed between the free-text drug descriptions and those associated with NDC values that were transmitted in 
our sample of e-prescriptions. Incorrect mapping of the NDC identifier to the free-text drug description by unauthorized personnel, either at the e-prescribing technology vendor or at the end-user practice site, is one possible source. Another possible source of variance is the inability of some e-prescribing systems to delink the "back end" NDC numbers added by the e-prescribing application when prescribers make modification to their initial drug description selection. Finally, incorrect assignment of NDC values by drug manufacturers represents yet another possible source for the observed discrepancies. This may suggest the need for the FDA to take a more active role in the assignment and regulation of NDC numbers to drug products as opposed to such complete reliance on the drug manufacturers to correctly perform this task.

\section{Limitations}

Several limitations of this analysis should be noted. First, our sample was limited to e-prescriptions received at pharmacies of 1 national retail drugstore chain during a single day and may therefore not be representative of all e-prescriptions that are issued in the ambulatory care setting. This potential limitation is mitigated by the fact that e-prescriptions in the sample were issued by a large number of geographically dispersed prescribers using more than 500 different e-prescribing software applications. Second, we did not directly compare the 3 reference NDC database files that were used in the study nor did we access other commercially available drug databases to determine the full scope of differences in NDC coverage that may exist. Such a comparison, however, does represent a potential area of future study, as differences clearly exist.

Third, the investigators used only 1 of the drug compendia, albeit a widely used one (First Databank), to match the majority of the drug concepts based on NDC numbers and free-text drug descriptions in the e-prescription message. We do not know which drug compendium may have been used by prescribers' software applications to generate study e-prescriptions, and it is possible there were drug description variances between the different drug compendia.

\section{Conclusions}

The transmission of accurate and interpretable drug identifiers from prescribers to receiving pharmacies is essential to realize the full benefits of e-prescribing. At best, inaccurate drug identifiers can result in pharmacy workflow disruptions and inefficiencies. At worst, they may significantly threaten patient safety.

The NDC system is the most widely recognized and used drug identification system in e-prescribing, and our study found that most NDC values that are transmitted are valid. However, our analysis identified a number of limitations and shortcomings to the use of NDC numbers in e-prescribing. These included a large number of unrepresentative NDC numbers and a significant proportion for which the free-text drug description entered by the prescriber did not match that implied by the NDC number. Most troubling was the finding that in $0.21 \%$ of cases ( $>2$ prescriptions per 1,000 ) the 2 drug descriptions pointed to completely different drug concepts. If this result is representative of the approximately 1 billion e-prescriptions that are transmitted each year in the ambulatory care setting, it is possible that as many as 2 million e-prescriptions could be affected.

To ensure accurate transmission of drug identity, e-prescribing technology vendors must maintain complete and up-to-date drug database files in their systems and ensure that patient medication histories are assigned current NDC identifiers on a regular basis. They should also conduct regular validation checks to ensure that the drug description associated with the NDC identifier and the free-text drug description that is sent in the e-prescription message point to the same drug concept before it is transmitted to the pharmacy. For their part, all commercial drug compendia must ensure that the drug identifier mappings they provide are accurate and include detailed implementation guidance to e-prescribing technology vendors that subscribe to their products and services. Finally, our study suggests that the role of the FDA in assigning the NDC numbers should be reconsidered vis-à-vis exclusive reliance on the drug manufacturers for this important task.

\section{Authors}

AJIT A. DHAVLE, PharmD, MBA, is Vice President; STACY WARD-CHARLERIE, PharmD, is Clinical Informatics Pharmacist; and JOSHUA RUIZ, CPhT, is Quality Specialist, Clinical Quality, Surescripts, Arlington, Virginia. MICHAEL T. RUPP, PhD, is Professor of Pharmacy Administration, Midwestern University, Glendale, Arizona, and VISHAL P. AMIN, PharmD, is Senior Consultant, Quality Assurance, CVS Health, Woonsocket, Rhode Island.

AUTHOR CORRESPONDENCE: Ajit A. Dhavle, PharmD, MBA, Surescripts, 2800 Crystal Dr., Arlington, VA 22202. Tel.: 703.921.2149; Fax: 703.921.2191;

E-mail: ajit.dhavle@surescripts.com.

\section{DISCLOSURES}

Dhavle, Ward-Charlerie, and Ruiz are employees of Surescripts. Amin is an employee of CVS Health, and Rupp is an employee of Midwestern University. Rupp reports receipt of consulting fees from Surescripts during the conduct of this study. All other authors declare no conflicts of interest in the research.

The content in this article is solely the responsibility of the authors and does not necessarily represent the official view of Surescripts, CVS Health, or Midwestern University. 
Study design and concept were created by Dhavle, Ward-Charlerie, and Amin, along with Rupp and Ruiz. Dhavle, Ward-Charlerie, Amin, and Ruiz collected the data, along with Rupp. Data interpretation was performed by Dhavle, Ward-Charlerie, and Ruiz, along with Rupp and Amin. The manuscript was written and revised by Dhavle, Rupp, and Ward-Charlerie, along with Amin and Ruiz.

\section{ACKNOWLEDGMENTS}

We are thankful to Keith Fisher, Julie Suko, Reem Mohamed, Patrick McLaughlin, Steve Franko, Yuze Yang, Sara Juster, Richard Lee, Valentina Lengkong, and Jade Pressley for their help and assistance with this research study.

\section{REFERENCES}

1. Desroches CM, Agarwal R, Angst CM, Fischer MA. Differences between integrated and stand-alone e-prescribing systems have implications for future use. Health Aff (Millwood). 2010;29(12):2268-77.

2. Abramson EL, Barrón Y, Quaresimo J, Kaushal R. Electronic prescribing within an electronic health record reduces ambulatory prescribing errors. Jt Comm J Qual Patient Saf. 2011;37(10):470-78.

3. McKibbon KA, Lokker C, Handler SM, et al. Enabling medication management through health information technology. Evidence Report/ Technology Assessment No. 201. (Prepared by the McMaster University Evidence-based Practice Center under Contract HHSA 290-2007-10060-I.) AHRQ Publication No. 11-E008-EF. Rockville, MD: Agency for Healthcare Research and Quality. April 2011. Available at: http://www.ahrq.gov/clinic/ tp/medmgttp.htm. Accessed September 25, 2015.

4. Hollingworth W, Devine EB, Hansen RN, et al. The impact of e-prescribing on prescriber and staff time in ambulatory care clinics: a time motion study. J Am Med Inform Assoc. 2007;14(6):722-30.

5. Dhavle AA, Rupp MT. Towards creating the perfect electronic prescription. J Am Med Inform Assoc. 2015;22(e1):e7-12.

6. Teich JM, Osheroff JA, Pifer EA, Sittig DF, Jenders RA; CDS Expert Review Panel. Clinical decision support in electronic prescribing: recommendations and an action plan: report of the joint clinical decision support workgroup. J Am Med Inform Assoc. 2005;12(4):365-76.

7. Bell DS, O'Neill S, Reynolds K, Schoeff D. Evaluation of RxNorm in ambulatory electronic prescribing. TR-941-CMS. RAND Health. 2011. Available at: http://www.rand.org/content/dam/rand/pubs/technical_ reports/2011/RAND_TR941.pdf. Accessed September 25, 2015.

8. American Medical Informatics Association. Standards for medical identifiers, codes, and messages needed to create an efficient computer-stored medical record. J Am Med Inform Assoc. 1994;1(1):1-7.

9. National Council for Prescription Drug Programs. SCRIPT Standard Implementation Guide. Version 10.6. Scottsdale, AZ; October 2014

10. U.S. Food and Drug Administration. National Drug Code Directory. Available at: http://www.fda.gov/Drugs/InformationOnDrugs/ucml42438. htm. Accessed September 25, 2015.
11. Levinson DR. The Food and Drug Administration's National Drug Code Directory. Department of Health and Human Services, Office of Inspector General. August 2006. Available at: http://oig.hhs.gov/oei/reports/oei-06-0500060.pdf. Accessed September 25, 2015.

12. Simonaitis L, McDonald CJ. Using National Drug Codes and drug knowledge bases to organize prescription records from multiple sources. Am J Health Syst Pharm. 2009;66(19):1743-53.

13. Grossman JM, Cross DA, Boukus ER, et al. Transmitting and processing electronic prescriptions: experiences of physician practices and pharmacies. J Am Med Inform Assoc. 2012;19:353-59.

14. Halamka JD. The benefits of RxNorm. Life as a Healthcare CIO [blog]. November 2, 2011. Available at: http://geekdoctor.blogspot.com/2011/11/ benefits-of-rxnorm.html. Accessed September 25, 2015.

15. National Council for Prescription Drug Programs. SCRIPT Implementation Recommendations. Version 1.28. September 2014. Available at: http://www. ncpdp.org/NCPDP/media/pdf/SCRIPTImplementationRecommendations V128.pdf. Accessed September 25, 2015.

16. U.S. Department of Health and Human Services, Office of Inspector General. The FDA prescription drug file. OEI-03-90-02300. November 1991. Available at: http://oig.hhs.gov/oei/reports/oei-03-90-02300.pdf. Accessed September 25, 2015.

17. First Databank, Inc. FDB MedKnowledge [brochure]. 2012. Available at: http://www.fdbhealth.com/ /media/downloads/form\%20required/us/brochure\%20-\%20fdb\%20medknowledge.ashx. Accessed September 25, 2015.

18. National Library of Medicine. RxNorm technical documentation. January 1, 2012. Available at: http://www.nlm.nih.gov/research/umls/ rxnorm/docs/2012/rxnorm_doco_full_2012-1.html. Accessed September 25, 2015.

19. Light RJ. Measures of response agreement for qualitative data: some generalizations and alternatives. Psychol Bull. 1971;76(5):365-77.

20. Suko J. Navigating stormy seas in drug classification. Part Two: Management challenges of NDC reuse. KNOWHOW: THE FDB BLOG. October 30, 2013. Available at: http://www.fdbhealth.com/blog/2013/october/part-twomanagement-challenges-of-ndc-reuse/. Accessed September 25, 2015.

21. Odukoya O, Chui MA. Retail pharmacy staff perceptions of design strengths and weaknesses of electronic prescribing. J Am Med Inform Assoc. 2012;19(6):1059-65.

22. Warholak TL, Rupp MT. Analysis of community chain pharmacists' interventions on electronic prescriptions. J Am Pharm. Assoc. 2009;49(1):59-64.

23. Nanji KC, Rothschild JM, Boehne JJ, Keohane CA, Ash JS, Poon EG. Unrealized potential and residual consequences of electronic prescribing on pharmacy workflow in the outpatient pharmacy. J Am Med Inform Assoc. 2014;21(3):481-86.

24. Hincapie AL, Warholak T, Altyar A, Snead R, Modisett T. Electronic prescribing problems reported to the Pharmacy and Provider ePrescribing Experience Reporting (PEER) portal. Res Social Adm Pharm. 2014;10(4):647-55.

25. Odukoya OK, Stone JA, Chui MA. E-prescribing errors in community pharmacies: exploring consequences and contributing factors. Int J Med Inform. 2014;83(6):427-37. 